\title{
Characterization of the Linearity of InGaAs Photodetectors Using Series Resistance
}

\author{
Nassim Rahimi ${ }^{1}$, Arshey Patadia ${ }^{1}$, Davorin Babic ${ }^{1}$, Dragan Grubisic ${ }^{1}$, Johannes Kunsch ${ }^{2}$ \\ ${ }^{1}$ Laser Components Detector Group, 7755 S Research Dr. Tempe AZ 85284, USA, \\ 2 Laser Components GmbH, Werner-von-Siemens-Str. 15, 82140 Olching, Germany \\ nrahimi@laser-components.com, apatadia@laser-components.com,dbabic@laser-components.com, \\ dgrubisic@laser-components.com, j.kunsch@lasercomponents.com
}

\begin{abstract}
:
An economical technique has been developed to characterize photodiode linearity. The technique is based on measuring photodiode's series resistance and is designed for industry applications. The measurement set-up has been made very simple by using only a source-measure unit and plotting software. Presented results clearly show advantages and validity of the proposed technique for characterizing linearity of InGaAs photodiodes using series resistance.
\end{abstract}

Key words: nonlinearity, InGaAs, photodiode, photodetector, characterization

\section{Introduction}

Optical detectors with cut-off wavelengths from 1.7 to $2.6 \mu \mathrm{m}$ are becoming increasingly of interest because they have technologically important applications such as NIR-FTIR spectroscopy, laser monitoring, non-contact temperature measurements and fiber-optic communications [1].

$\ln (\mathrm{x}) \mathrm{Ga}(1-\mathrm{x})$ As photodiodes grown on $\ln \mathrm{P}$ substrates are widely used in the specified wavelength range. The $\ln (\mathrm{x}) \mathrm{Ga}(1-\mathrm{x})$ As material can be grown lattice-matched to the InP (having 1.7 $\mu \mathrm{m}$ cut-off wavelength) or latticemismatched to the InP using buffer layer with cut-off wavelengths covering the range from 1.9 to $2.6 \mu \mathrm{m}$ [2]. Mismatched $\ln (\mathrm{x}) \mathrm{Ga}(1-\mathrm{x})$ As devices have poor optical and electrical performances unless a buffer layer is engineered into their structure to prevent propagation of mismatch generated dislocations into the active layer. Most applications require low noise (low dark current) operation which is strongly compromised by the dislocations acting as generation-recombination centers.

Detector linearity is another critical performance parameter besides the well-known low dark current. The linearity is required for accuracy of the electrical response of the detectors as the incident light intensity increases. In addition, the linearity enables the use of the InGaAs photodiodes at various power levels. Many industries where the detectors will be used require high measurement precision so the linearity of the detectors must be clearly demonstrated. In some applications, accuracy is so critical that each detector has to be tested and experimentally verified for linearity. In these cases, a simple and reliable measurement to characterize linearity of a detector is very useful and critical

A photodiode is defined to be linear when its photoelectric conversion efficiency (responsivity) is independent of the incident optical power. Each photodiode behaves as a linear detector for a certain range of optical power and becomes nonlinear for higher intensity optical power, the nonlinearity threshold being a characteristic of the particular photodiode.

The nonlinearity of a photodiode is a function of several factors like generated photocurrent, incident optical power, the ambient temperature, etc. However, the series resistance of the photodiode appears to be the most important factor in determining the linearity. One also needs to mention the incident beam diameter because it has been observed it has an influence on the linearity, but in a different manner for various types of photodiodes, however the influence is not wavelength dependent [3].

As an example of the importance of photodiode detector linearity, Fourier Transform Infrared Spectroscopy (FTIR) is especially sensitive to the nonlinearity of the detectors [4]. The mathematics of the transformation requires the linearity of the measured data. Artificial features 
appear in the spectra if the linearity criterion is not satisfied. A typical nonlinearity induced feature in the FTIR spectra is observation of non-vanishing spectral response in the wavelength region beyond the cut-off wavelength of the detector, frequently accompanied by a dip (called "foot") at the detector cut-off. The linearity of a photodiode detector is generally characterized by measuring diode response to optical signals of varying intensities.

In the conventional optical linearity range measurement mostly beam-addition method is used [1]. In this method the measured signal of two beams is combined and compared with the sum of the signals measured separately for the same two beams. A typical configuration for the beam-addition measurement is in Figure 1 (from Ref.1). The measurement usually involves a calibrated light source with a set of optical fibers or free space optics in addition to the instrument that measures the diode photogenerated signal $[1,5,6]$. A typical light source to be used in the measurement is a laser diode with the control electronics including a purpose-built low-noise current driver. The temperature of the laser diode is needed to be controlled and stabilized with a cooler. The laser frequency is required to be tuned over a specific range. The beam-addition measurement setups also include a collimating lens mounted on lens-positioning instruments and multiple apertures to reduce the sidebeams and stray light. The measurement configuration includes a set of neutral density filters to control the laser light intensity. In the beam-addition measurements, the laser beam is commonly divided into two approximately equal parts with a cube beamsplitter. In the particular setup shown in Figure 1, two iris diaphragms are placed after the beamsplitter to reduce the stray light and the sidebeams again. The optical system of the beam-addition measurement should be aligned first using one beam coming from the beamsplitter to strike the center of the photodiode and then the second beam should be adjusted with a plane mirror to coincide with the first beam and create an interference pattern on the detector. The accuracy of the two beams positioned on top of each other can be verified with a CCD camera. The photogenerated current will then be measured for (a) the first beam, (b) the second beam and (c) both beams together using a highperformance digital multimeter or a current-tovoltage converter and a multimeter.

The nonlinearity is defined by its change as

$\Delta N L=1-\frac{I_{A}+I_{B}}{I_{C}}$

where $I_{A}$ and $I_{B}$ denote the photogenerated currents caused by the two separate beams and and $\mathrm{I}_{\mathrm{C}}$ denotes the photogenerated current caused by the combined beam [1].

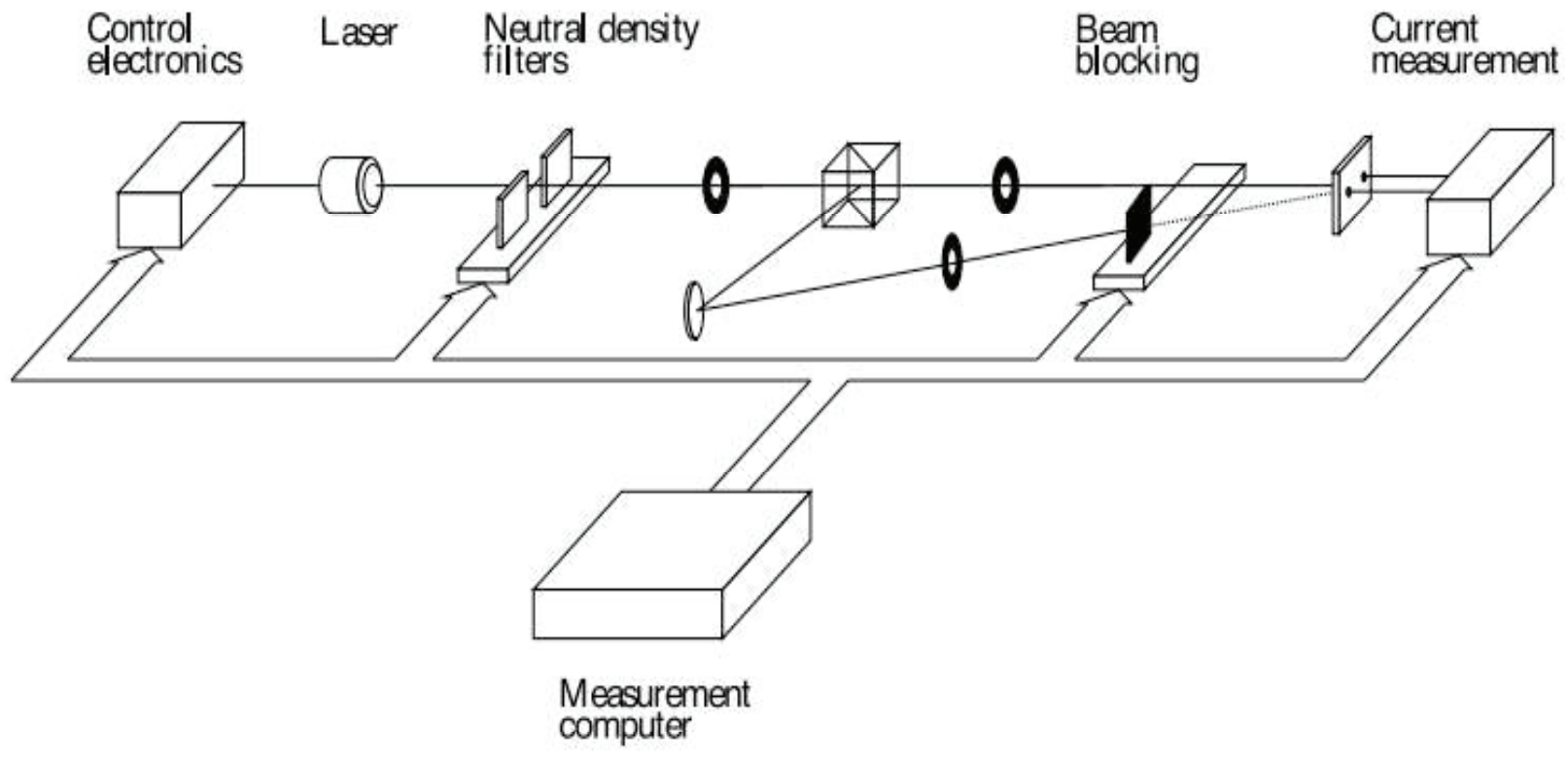

Fig.1. The automated measurement set-up used in the linearity measurement [1]. 


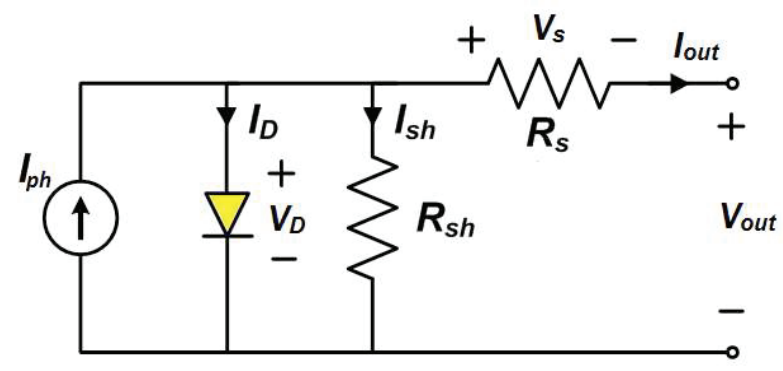

Fig. 2. Photodiode equivalent circuit under illumination.

The optical techniques for characterizing photodiode nonlinearity are relatively complicated and need lots of electrical and optical equipment. We propose another technique to characterize the nonlinearity range of a photodiode that does not require any optical components and instruments.

Relative simplicity of the proposed technique makes it more suitable for a production environment where fast and easy testing of devices is at premium. The proposed technique is based on fundamental physics of the optical response of a photodiode detector.

At the optical power levels involved, the nonlinear response effects are caused by 'sinking' of a portion of the photogenerated current within the photodiode itself. The 'sinking' is caused by a slight forward bias of the photodiode induced by the voltage drop across its series resistance as shown in Figure 2. The Figure 2 shows the photodiode equivalent circuit in sufficient detail to explain the origin of the nonlinearity. The series resistance $R_{s}$ usually arises from the contacts and the wire bonds of the photodiode. Nonlinear form of the forward biased I-V diode characteristics (Eq. 2) makes the 'sinking' nonlinear thus causing the nonlinear relationship between the photodiode output current $\mathrm{I}_{\text {out }}$ and the photogenerated current $\mathrm{I}_{\mathrm{ph}}$ which is the actual cause of the photodiode's nonlinear optical response.

$$
I=I_{0}\left(\exp \frac{q V}{n K T}-1\right)
$$

For the same photogenerated current values, lower series resistance values cause lower forward bias of the photodiode thus directing more photogenerated current to the output and keeping the optical response linear. Thus, the series resistance of a photodiode can be used to characterize its optical linearity and/or nonlinearity.
We estimate the series resistance value from measured forward biased I-V characteristics of a particular diode in the dark making this technique simpler than the usual optical measurements since no optical components are required.

\section{Experimental Setup}

The series resistance of a photodiode is estimated from its forward biased I-V curve measured using the experimental setup shown schematically in Figure 3.

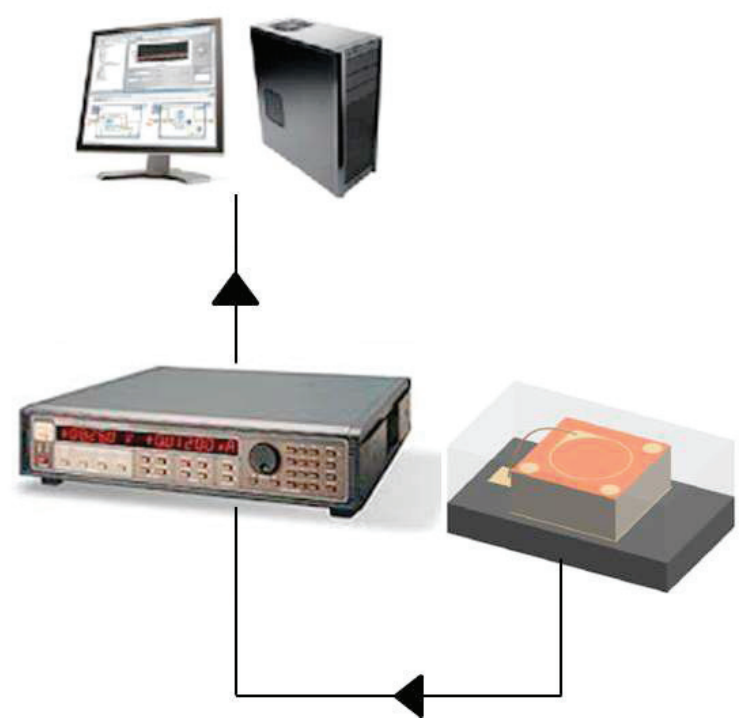

Fig. 3. I-V measurement setup schematic.

The simplicity of this setup makes it very desirable involving just a source-measure unit such as a Keithley 237 that applies current and measures the resulting voltage and a computer software to collect data and plot I-V curve.

FTIR spectra of several photodiodes to illustrate both linear and nonlinear responses are presented below together with results of the series resistance estimations for the diodes that support the FTIR results.

Figure 4 shows FTIR spectra of several Laser Components photodiodes. The spectra of two photodiodes show monotonic transition to straight tails with small noise; however, the spectra of the third photodiode (marked "red") displays a small, but clearly noticeable "foot" accompanied by a slopped noisy line at wavelengths beyond the detector cut-off. These results indicate that the first two photodiodes are linear while the third photodiode is nonlinear for the power levels used in the measurements. Per the discussion of the influence of the series resistance of the linearity, the FTIR results also suggest that the series resistances of the first two photodiodes should be lower than the 
series resistance of the third photodiode. Determination of the series resistance values by measuring the forward biased I-V curve of the diodes and performing the curve fitting is outlined below.

Figure 5 illustrates the series resistance value estimation steps, while Table I presents the estimated series resistance values for the photdiodes whose spectra are shown in the Figure 4 and a few additional detectors. The series resistances of the photodiode devices are estimated by the following steps:

1. The forward biased I-V curve for each device is measured.

2. The logarithm of current $(\log (I))$ versus voltage $(\mathrm{V})$ is plotted. The ideal diode I$\checkmark$ curve is exponential so that the $\log (\mathrm{I})$ versus $\mathrm{V}$ curve should be linear, however, in the non-ideal case, at higher voltages the diode current does not exponentially change as the voltage increases and the $\log (\mathrm{I})-\mathrm{V}$ curve deviates from linear line due to the series resistance effect.

3. A tangent line of the linear part of $\log (\mathrm{I})-\mathrm{V}$ is drawn.
4. A horizontal (fixed current) line is drawn where the current does not exponentially change as the voltage increases.

5. We record the current corresponding to the intersection of the two lines $\left(I_{s}\right)$.

6. The two voltage values are needed to calculate the series resistance. One of them $\left(V_{1}\right)$ is taken at the intersection of the two lines (where the $\mathrm{I}_{\mathrm{s}}$ is registered) and the second voltage point $\left(V_{2}\right)$ is taken at the point where the horizontal line intersects the Log(I)-V curve.

7. Series resistance is calculated based on the conventional Ohm law:

$$
R_{s}=\frac{\Delta V}{I_{s}}=\frac{\left(V_{2}-V_{1}\right)}{I_{s}}
$$

Since the series resistance values are obtained from the curve fitting, the error of $\pm 0.1 \Omega$ is estimated for the series resistances.

The series resistance values from the Table I correlate well with the observed linearity of the detectors shown in Figure 4.

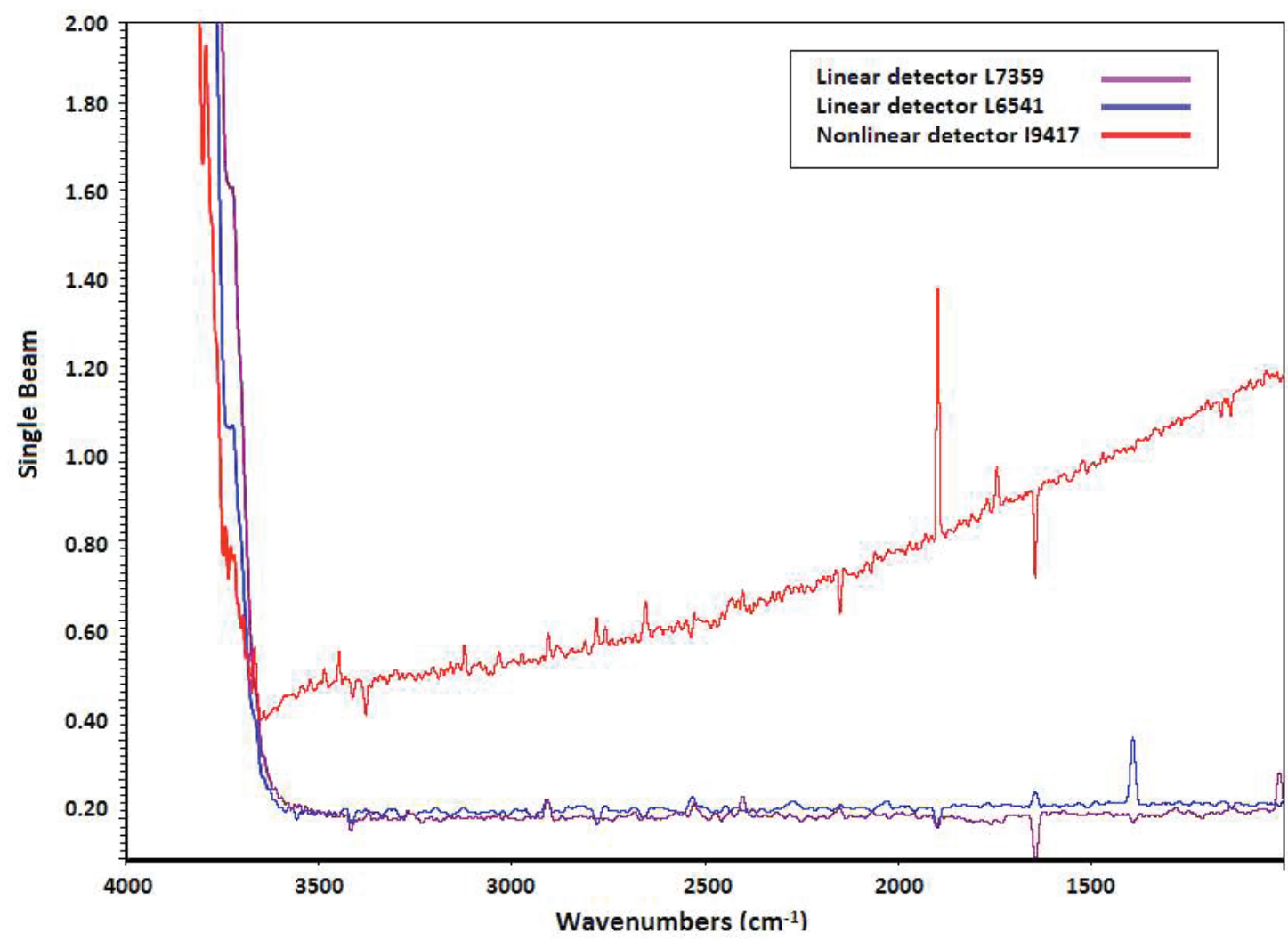

Fig.4. FTIR spectra for linear and nonlinear photodiodes showing "foot" and sloped line beyond the detector cutoff (for nonlinear detector). 


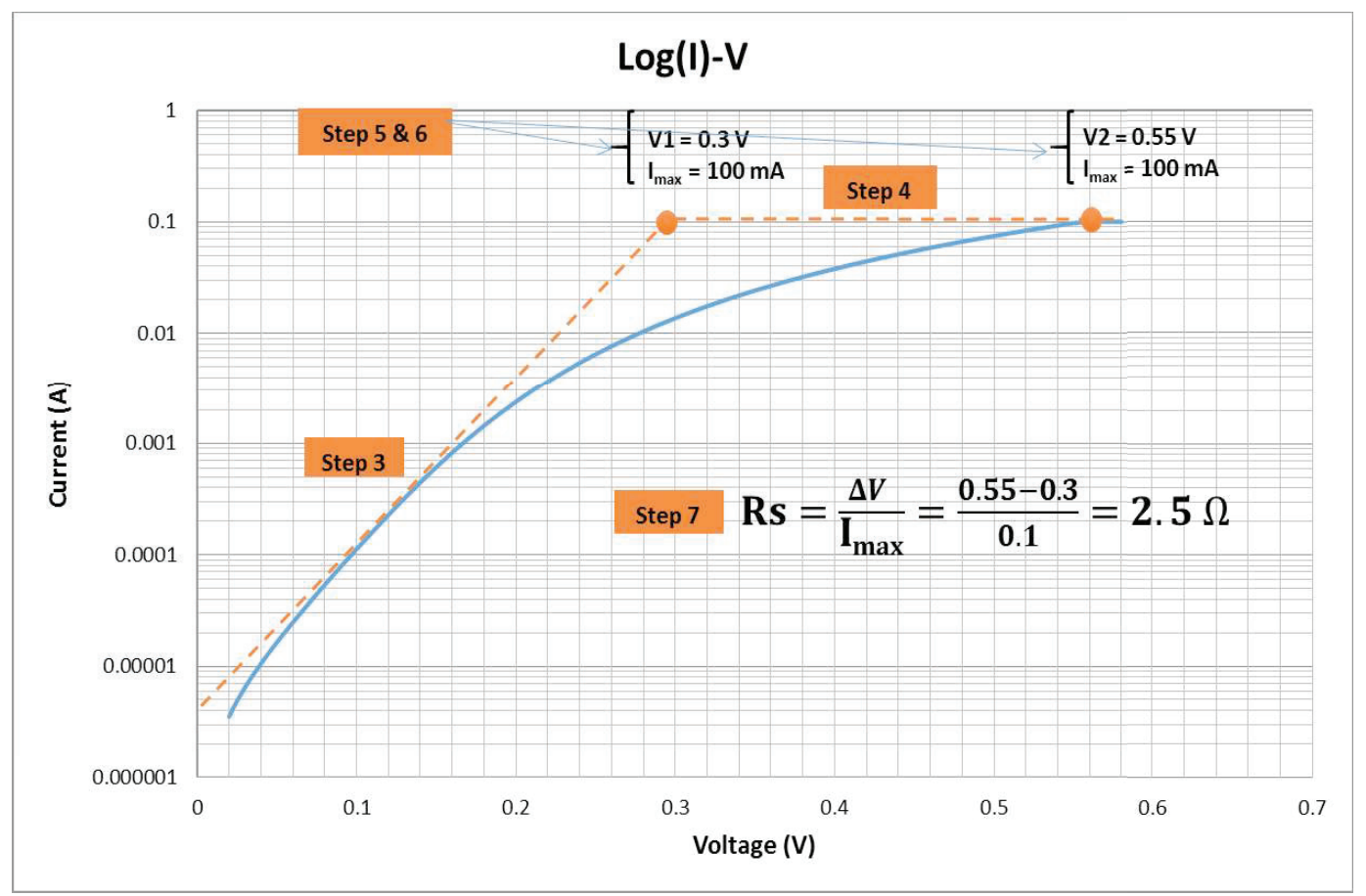

Fig.5. An illustration of the series resistance value estimation steps after measuring the forward bias I-V curve up to $100 \mathrm{~mA}$ and plotting the logarithm of current vs. voltage.

Tab. 1: Series resistance values for different tested IG26X1000T9 detectors.

\begin{tabular}{|c|c|}
\hline Device Serial No. & $\begin{array}{l}\text { Series } \\
\text { Resistance }(\boldsymbol{\Omega})\end{array}$ \\
\hline L7359-IG26X1000T9 & 1.5 \\
\hline L6541-IG26X1000T9 & 2.5 \\
\hline L1986-IG26X1000T9 & 2.3 \\
\hline F1346-IG26X1000T9 & 1.9 \\
\hline I9417-IG26X1000T9 & 6.1 \\
\hline
\end{tabular}

The nonlinear device has by far the largest series resistance, above $6 \Omega$, while the linear detectors have series resistance values from $1.5 \Omega$ to $2.5 \Omega$. The above results clearly show validity and advantages of the proposed technique for characterizing linearity of InGaAs photodiodes using series resistance.

\section{Conclusions}

Linearity of a photodetector is a critical performance parameter because the linearity is required for accurate electrical response of the photodetector as the incident optical power increases. Usual techniques of characterizing the linearity are demanding necessitating a number of aligned optical components and sources besides usual electrical components. An economical and simple technique to characterize the linearity of InGaAs photodiodes is being presented in this work.
The technique is suitable to many industrial applications. It is based on the fundamental relation between the linearity of a photodiode and its series resistance. The technique involves measuring the forward biased I-V characteristics of a photodiode and applying the collected data to estimate the series resistance value. Series resistances of several photodiodes have been evaluated by the above procedure and successfully correlated with their FTIR spectra. The above results clearly show validity and advantages of the proposed technique for characterizing linearity of InGaAs photodiodes using series resistance

\section{References}

[1] A. Haapalinna, T. Kubarsepp, P. Karh, E. Ikonen, Meas. Sci. Technol. 10,1075-1078 (1999).

[2] J. Piotrowski, J. Kaniewski, IEE Proc.Optoelectron.146, 173-176 (1999).

[3] L. P. Boivin, Automated absolute and relative spectral linearity measurements on photovoltaic detectors Metrologia 30, 355-60 (1993).

[4] P. R. Griffiths, J. A. De Haseth, Fourier Transform Infrared Spectroscopy, Wiley, 2nd ed. (2007).

[5] T. L. Paoli, IEEE J. of Quant. Electron. QE-16, 340-346 (1980).

[6] A. Ramaswamy, N. Nunoya, M. Piels, L. A. Johansson, L. A. Coldren, J. E. Bowers, International Topical Meeting on Microwave Photonics, October 14-16, Valencia (2009). 